\title{
Research on the Integration of Prefabricated Building and BIM Based on Bibliometrics
}

\author{
WANG Qiuyu ${ }^{1, \mathrm{a}}$, GONG Zhiqi ${ }^{1,2 * b}$ \\ ${ }^{1}$ School of Civil Engineering, Qinghai University \\ ${ }^{2}$ Qinghai Provincial Key Laboratory of Energy-saving Building Materials and Engineering Safety Xining, Qinghai, China
}

\begin{abstract}
Analyzing the literature in the field of prefabricated building and BIM integration is helpful to promote the development of prefabricated building informatization. Using the Web of Science core collection database as the data source, this study screened relevant literatures at home and abroad from 2000 to 2020 . CiteSpace and VOSviewer were used to conduct visual analysis on the characteristics of published articles, research hotspots and evolution trends based on bibliometrics. The results show that the related literature heat in this field will continue to increase in China in the future. Design and management have become the focus and trend of current scholars in this field. Exploring all kinds of development obstacles has become a new research hotspot in the past two years.
\end{abstract}

\section{Introduction}

In recent years, with the support of central and regional policies, prefabricated buildings have developed rapidly in China. In 2016, the State Council issued a statement stating that prefab buildings should account for 30 percent of new construction space in the next 10 years ${ }^{[1]}$. In 2020 , multiple departments of the central government jointly issued a document, indicating that the development of prefabricated buildings plays an important role in the construction of intelligent construction industrial system. However, in the development process of prefabricated buildings, the traditional two-dimensional information guidance construction has been unable to solve the problems such as deepening design economically and effectively, information management, on-site hoisting, complex node processing, etc. ${ }^{[2]}$.

The concept of Building Information Modeling (BIM) has been very hot in construction projects in recent years [3]. With its advantages of digitalization and informatization, BIM technology provides new ideas and methods for solving the information occlusion in the whole life cycle of buildings. Through the integration of prefabricated building and BIM, the design efficiency of prefabricated standardized components can be improved and errors can be reduced in the design stage. In the construction stage, the site layout can be optimized and quality can be effectively supervised and managed. Information management can be carried out in the followup operation and maintenance stage ${ }^{[4]}$.

In the field of integrated research on prefabricated buildings and BIM, there is a lack of literature that applies bibliometric method to deeply explore relevant fields in China at present. Based on this, this study adopts the method of combining bibliometrics and visual analysis to sort out 130 high-quality academic articles and analyze the research status of the integrated application of prefabricated buildings and BIM, in order to provide reference for subsequent studies.

\section{Research Methods and Data Sources}

\subsection{Research Methods}

In this study, quantitative analysis and qualitative analysis are combined to reveal the research hotspots and development trends of prefabricated building and BIM integration more comprehensively [5]. In terms of quantitative analysis, visual analysis of bibliometrics was carried out based on CiteSpace and VOSviewer knowledge graph software. In terms of qualitative analysis, this paper will analyze and sort out the research situation on the integration of prefabricated building and BIM according to the classical literature and knowledge graph information, and put forward development suggestions.

In terms of software application, CiteSpace literature analysis software was developed by Professor Chen Chaomei from Drexel University in the United States. Based on the Java environment platform, it can transform massive literatures into vivid and intuitive knowledge map [6]. VOSviewer is a visual analysis tool jointly developed by Nees Jan van Eck from Leiden University in the Netherlands and his colleagues, which is mainly used to analyze the coupling network, cooperative network, cocitation network and topic co-occurrence network of literature ${ }^{[7]}$. In this study, VOSviewer was used to analyze and draw institutional cooperative network, national distribution network and cooperative network among

ae-mail: dreamy46@foxmail.com

b*e-mail: gzhq2007@ foxmail.com 
authors. CiteSpace software is used to analyze the cooccurrence of keywords and the emergence of research hotspots.

\subsection{Data Sources}

The data in this study came from the core collection of Web of Science, and a comprehensive and in-depth analysis of relevant literature was conducted by using CiteSpace and VOSviewer software. Since the domestic research on the integration of prefabricated buildings and BIM appeared around 2000, the time span is set as 20002020.

In the core database of Web of Science, a total of 1464 related literatures were obtained by advanced search with the "prefabricated construction" or "prefabricated building" as the main topic. With "BIM" or "Building Information Modelling" as the main topic, 48,706 relevant literatures were obtained by advanced retrieval. By combining the two topics and eliminating the literatures with less relevance and those in other fields, 130 representative effective literatures with higher academic level were finally screened out. The literature retrieved and screened will be used as the main basis for the integrated research and analysis of prefabricated building and BIM. Data sources are shown in Table 1.

\section{Literature Publication Trend}

The quantitative analysis of literature distribution in the field of prefabricated buildings can objectively reflect the research status, research level and research scope, and then have an overall understanding of the research situation, discipline scale and research progress of this topic ${ }^{[8]}$.

\subsection{Statistical Analysis of literatures}

Statistical analysis was conducted on the number of published literatures from 2000 to 2020 to obtain the number of literatures in the field of integrated research on prefabricated buildings and BIM each year. According to the number of published papers each year and the increase over the years, the distribution map of the number of published papers and the increase over time was drawn, as shown in Figure 1.

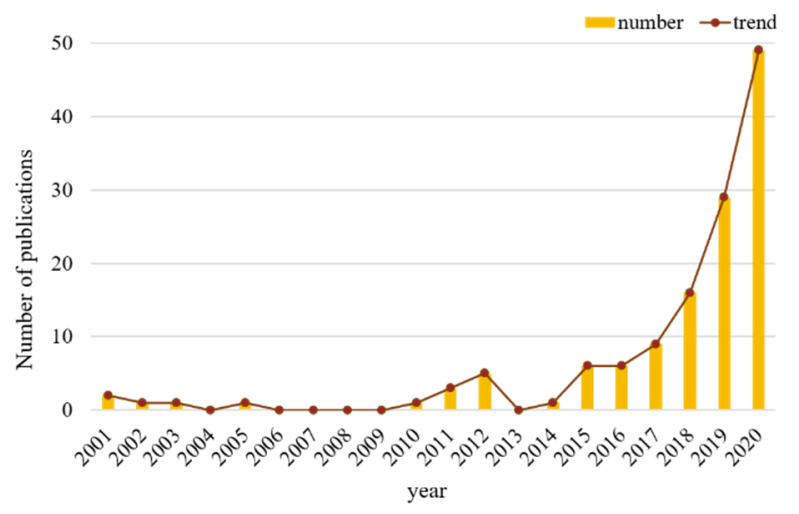

Figure 1. Annual publications from 2000 to 2020

As can be seen from Figure 1, the research on the integration of prefabricated buildings and BIM started relatively late in China. From 2001 to 2010, there was almost no relevant research, which was in the "blank period" of publication. From 2011 to 2015, some scholars began to pay attention to the research field of prefabricated building and BIM integration, which was in the "fluctuating period" of publishing papers. Around 2016, China's research literature on the integration of prefabricated buildings and BIM began to increase year by year, and there is an explosive growth, so 2016 is now in the "growth period" of publication.

Exploring the changing characteristics of these three development periods, we can find that it is closely related to the driving effect of policy. In August 2015, the ministry issued the industrialized building evaluation standard decided to comprehensive promotion prefabricated construction since $2016^{[9]}$, released in November the same year the ministry and the construction industry modernization development compendium ${ }^{[10]}$, prefabricated construction plan to 2020 accounted for more than $20 \%$ of the new buildings, this directly led to the 2016 prefabricated building with BIM number show explosive growth, integration research highlights the leading role of policy documents. From 6 papers in 2016 to 49 in 2020, the number of published papers has increased by 8 times. According to this development trend, the number of relevant domestic papers in this field will continue to increase in the next few years.

\subsection{Distribution Analysis of Institutions}

In order to analyze the distribution of major research institutions in the field of integrated research on prefabricated buildings and BIM, the number of published papers, the number of cited papers and the cooperation intensity of the institutions were statistically analyzed. The ranking of major research institutions is shown in Table 1.

Table 1. Statistics of main research institutions

\begin{tabular}{|c|c|c|c|c|}
\hline Number & Organization & Documents & Citations & $\begin{array}{c}\text { Total link } \\
\text { strength }\end{array}$ \\
\hline 1 & $\begin{array}{l}\text { hong kong } \\
\text { polytech } \\
\text { univ }\end{array}$ & 11 & 336 & 20 \\
\hline 2 & $\begin{array}{l}\text { shenzhen } \\
\text { univ }\end{array}$ & 8 & 196 & 14 \\
\hline 3 & $\begin{array}{l}\text { univ hong } \\
\text { kong }\end{array}$ & 8 & 358 & 13 \\
\hline 4 & $\begin{array}{c}\text { univ } \\
\text { melbourne }\end{array}$ & 6 & 115 & 4 \\
\hline 5 & deakin univ & 5 & 23 & 4 \\
\hline 6 & tongji univ & 5 & 6 & 2 \\
\hline 7 & $\begin{array}{l}\text { harbin inst } \\
\text { technol }\end{array}$ & 4 & 72 & 6 \\
\hline 8 & $\begin{array}{l}\text { guangzhou } \\
\text { univ }\end{array}$ & 4 & 63 & 4 \\
\hline 9 & $\begin{array}{l}\text { chongqing } \\
\text { univ }\end{array}$ & 4 & 72 & 3 \\
\hline 10 & $\begin{array}{c}\text { southeast } \\
\text { univ }\end{array}$ & 4 & 5 & 1 \\
\hline
\end{tabular}

From 2000 to 2020 , among the top 10 research institutions in the field of prefabricated building and BIM integration, The Hong Kong Polytechnic University published the most papers, with 11 papers published and 336 citations. It also had the closest cooperation with other 
institutions. Shenzhen University came in second with eight articles. The other research institutions are, in order, the University of Hong Kong, the University of Melbourne, Deakin University, Tongji University, Harbin Institute of Technology, Guangzhou University, Chongqing University, and Southeast University.

As can be seen from Table 2, some well-known universities constitute the main research groups in this field, and have made important technical support and theoretical research for its development. However, there is still a lack of research in this field in universities and enterprises, and the research depth is not enough or the emphasis is not enough, which is related to universities' preference for theory and enterprises' preference for practice.

\subsection{Co-occurrence Analysis of Authors}

The analysis of co-occurrence of authors is the exploration of collaborative network of authors, which can reflect the cooperation and communication between different authors [11]. The analysis type was set as "Co-authorship", the analysis unit as "Authors", the calculation method was "Full counting", the minimum frequency was set as " 1 ", and the VOSviewer was used for plotting. The network of co-authorship authors is shown in Figure 2.

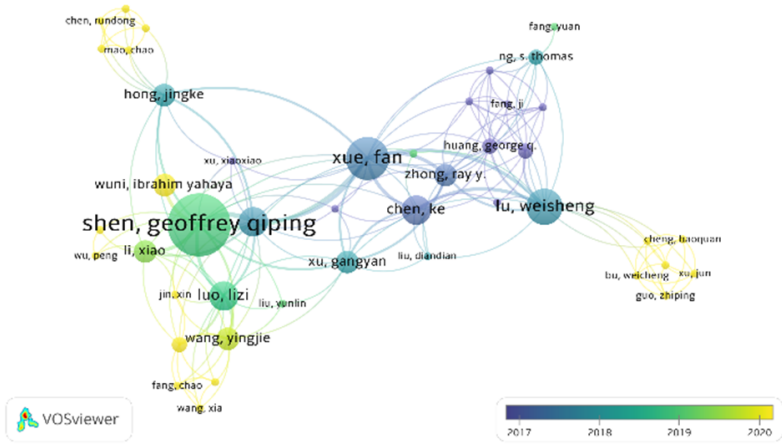

Figure 2. Network of co-authorship authors

As can be seen from Figure 2, there are many authors co-appearing in the research field of prefabricated building and BIM integration, and a cluster group with Shen Geoffrey Qiping, Xue Fan, Lu Weisheng and others as the center is formed. It is found that they are scholars with great influence in this field. Taking Shen Geoffrey Qiping as an example, the clustering scale of this group is the largest, which indicates that there is close cooperation between Shen Geoffrey Qiping and several other scholars. The connection also indicates that there are many cooperation and exchanges among the surrounding scholars, and a healthy and stable academic research group has been formed. Groups such as Wuni Ibrahim Yahaya and others are small, but their research is relatively new and may have more impact on the field in the future.

In general, the in-depth research in the field of assembly building and BIM integration has begun to take shape and developed well. The researchers who have published more papers are closely connected, forming their own cluster groups. The scattered scholars should cooperate and communicate more with other scholars. The scholars who form the scale of the cluster should strengthen cooperation, innovate content, and jointly promote the healthy and long-term development of prefabricated building and BIM technology.

\section{Analysis of Research Hotspots}

\subsection{Co-occurrence Analysis of Keywords}

Through the keyword co-occurrence analysis, it can better dig out the matching research hotspots in this field. Each keyword corresponds to a node, and the measurement of the connection effect of network nodes in the overall network is the centrality of nodes. The higher the centrality, the larger the nodes are, and the easier it is to become a research hotspot ${ }^{[12]}$. In the parameter setting of CiteSpace, the analysis type was selected as "Keyword" to conduct visual analysis. The knowledge graph of co-appearing keywords in the integrated research field of prefabricated building and BIM is shown in Figure 3.

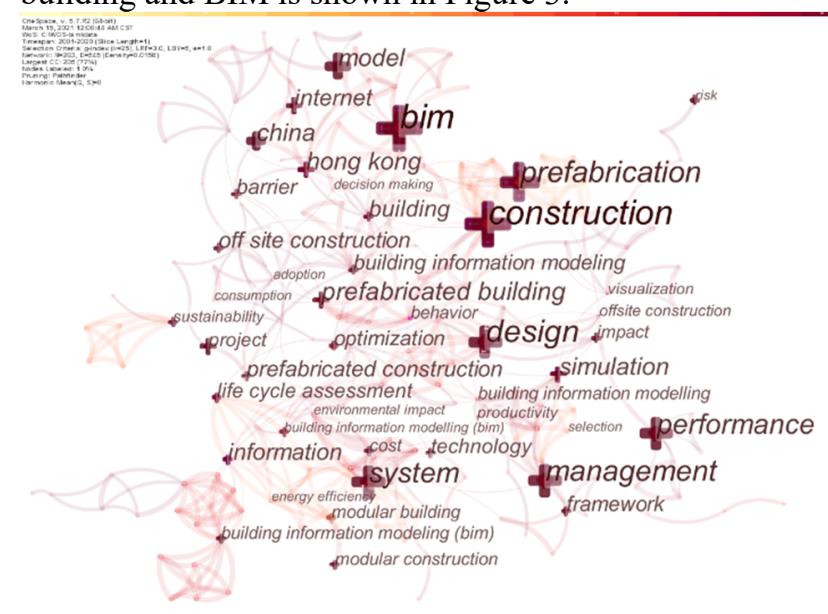

Figure 3. Network of keywords co-occurrence

According to the ranking results of word frequency and centrality, keywords such as "BIM", "construction", "design", "prefabrication" and "management" appeared the most frequently, excluding the search keywords "BIM", "prefabricated building" and "prefabricated construction". It can be found that "design" and "management" have been the research hotspots in this field in the past 20 years.

In the field of integrated research of prefabricated building and BIM, researchers tended to study technology in the early stage, but up to now, design and management have become the focus and trend of current scholars' research. In addition, it can be found from the figure that the frequency distribution of the occurrence of high frequency words in research hotspots is quite different and the research hotspots are relatively scattered, which indicates that multi-direction integrated research has become normal in this field.

\subsection{Keywords Analysis of Citation Bursts}

In the process of disciplinary research, the change of vocabulary frequency in the research content can often reflect the dynamics of the frontier of the research. In CiteSpace, emergences with a high rate of frequency 
change are generated through emergences detection to identify the frontier of disciplinary research, which has the characteristics of dynamic and comprehensive ${ }^{[13]}$. Built-in software algorithm was used to detect the emergent words, and the emergent words were obtained, as shown in Figure 4.

According to the intensity and time period of emergence, it is found that "barrier", "Modular Building", "BIM", "information", "optimization" and other aspects are the latest trend in the research field of assembly building and BIM integration. Among them, "barrier" has become the word with the highest emergence strength in the past two years, indicating that obstacles encountered in the research and development process in the field have become the most concerned issue for scholars today. Although "Modular Building" came in second among the top 10 in intensity, it lasted the longest (2012 - 2020), indicating that modular building is the core and most persistent research topic in this field.

\begin{tabular}{lllll}
\multicolumn{5}{c}{ Top 10 Keywords with the Strongest Citation Bursts } \\
\multicolumn{1}{c}{ Keywords } & Year Strength Begin End \\
barrier & 2001 & $\mathbf{2 . 6 9} 2019$ & 2020 \\
modular building & 2001 & 1.83 & 2012 & 2020 \\
building information modelling (bim) & 2001 & 1.69 & 2018 & 2020 \\
information & 2001 & 1.69 & 2012 & 2020 \\
optimization & 2001 & 1.61 & 2018 & 2020 \\
hong kong & 2001 & 1.55 & 2016 & 2020 \\
offsite construction & 2001 & 1.5 & 2018 & 2020 \\
productivity & 2001 & 1.5 & 2018 & 2020 \\
behavior & 2001 & 1.33 & 2016 & 2020 \\
renewable energy & 2001 & 1.28 & 2012 & 2020 \\
\hline
\end{tabular}

Figure 4. Top 10 keywords with the strongest citation bursts

\section{Conclusions}

Based on the Web of Science core collection database from 2000 to 2020, this paper uses CiteSpace and VOSviewer knowledge map software to analyze the development situation and research hotspots of 130 literatures, and the research conclusions are as follows:

From the perspective of the number of published papers, the number of published papers in the field of integrated research on prefabricated buildings and BIM has seen an explosive growth after 2016, highlighting the leading role of policy documents. In the future, relevant domestic literatures in this field will continue to increase. In terms of research institutions, well-known universities constitute the main group of research institutions in this field, and have made important technical support and theoretical research for its development, while ordinary universities and companies still lack research in this field. In terms of researchers, researchers who publish more papers are closely connected with each other, forming their own cluster groups, and scattered scholars should carry out more cooperation and communication with other scholars.

From the perspective of research hotspots, China lays more emphasis on the basic theoretical research in the past. According to the current situation, design and management have become the focus and trend of current scholars in this field, which shows that prefabricated building with BIM integration application direction has gradually extended to the design and management direction. In addition, the research hotspots are also scattered, which indicates that the integration research of multiple directions has become normal. In the future, more and more integration research of multiple fields and multiple directions will emerge in this field.

From the perspective of evolution trend, the research on the integration of prefabricated building and BIM gradually develops from the technical level to the management direction, and the exploration of various development obstacles has become the focus and trend of various scholars in the past two years. However, while focusing on management, China's prefabricated building and BIM integration technology are still progressing steadily, and modular building has been the most core and lasting research topic in this field. Technology is the core of management. Therefore, technology and management should go hand in hand, the combination of theory and practice, in order to effectively promote the development of prefabricated building information.

The deficiencies of this study are as follows: This study only uses the journal literature data of the core collection database of Web of Science as the data source, and does not analyze the CNKI database. As a result, the domestic analysis is not targeted enough. In the future, the literature data of different databases can be combined for comparative analysis. In addition, the number of the selected 130 literatures is small and the time-span is long. In the future, the research period can be narrowed down to focus on the development of each period to provide development suggestions for the integrated research field of prefabricated buildings and BIM.

\section{References}

1. General Office of the State Council. No.71 Document [S]. 2016.

2. He Wenyu, Wen Xinpeng, Huang Xinkai, Peng Junlong, Liao Ling. Academic hotspots and evolving trends of integrated research on prefabricated buildings and BIM -- A visual analysis of bibliometrics based on CiteSpace and UCINET [J/OL]. Information Technology in Civil and Architectural Engineering, 211, 1-11.

3. Wang Ting, Xie Zhaoxu. BIM literature analysis based on four English core journals from 2004 to 2014 [J]. Journal of Engineering Management, 2016,30 (1): 37-42.

4. Liu Bo. Application of BIM technology in prefabricated building process $[\mathrm{J}]$. Green Environmental Building Materials,2020(6):186-187.

5. CHEN Bin. Research on Visualization of Lean Construction Field Based on Knowledge Graph [D]. Hefei University of Technology,2019.

6. CHEN C . CiteSpace II: detecting and visualizing emerging trends and transient patterns in scientific literature $[\mathrm{J}]$. Journal of the American Society for Information Science and Technology,2006,57(3) :359-377 . 
7. Li J. Principles and Applications of Scientific Knowledge Graph: A Guide to VosViewer and Citnetex - plorer [M]. Beijing: Higher Education Press, 2018.

8. Hou Haiyan, Liu Zeyuan, Luan Chunjuan. Quantitative analysis of international science metrology research frontier based on knowledge graph [J]. Science Research Management, 2009, 30(1): 164-170.

9. Ministry of Housing and Urban-Rural Development, PRC. Evaluation standard for industrial buildings: GBT51129-2015 [S]. Beijing: China Architecture and Building Press, 2015.

10. Construction industry modernization development outline [Z]. 2016.

11. Chen Yue, Chen Chaomei, Liu Zeyuan, et al. The Methodological Function of CiteSpace Knowledge Graph [J]. Studies in Science of Science, 2015,33 (2): 242-253.

12. Zhao Rongying, Yu Kaipeng, Chen Bikun. Comparison of Visualization and Analysis of Domestic and Foreign Factors Research [J]. Information Science, 2014,35 (3): 3-11.

13. Zhang Yongcheng, Liu Aohan, Zhong Botao. A bibliometric analysis of construction economy based on CiteSpace [J]. Building Economics, 2020,41(S1):50-55. 